\title{
Performance Analysis of Eye localization Methods for Real Time Vision Interface using Low Grade Video Camera
}

\author{
Krupa Jariwala \\ Assistant Professor \\ Computer Engineering Department \\ SVNIT, Surat
}

\author{
Upena Dalal, Ph.D. \\ Associate Professor \\ Electronics Engineering Department \\ SVNIT, Surat
}

\begin{abstract}
It is necessary to correctly and precisely achieve eye localization, which is a fundamental step for the initialization for other eye localization based applications. There are various methods including special equipment based methods and image based methods to perform this task. Special equipment based methods are very accurate but not practical for day to day use. Image based approaches are user friendly, allows free head movement, avoids specialized hardware and infrared exposure but more difficult to implement. Performance is analysed for state of the art eye localization methods for real time vision interface using low grade camera that use similar objective criterion for error measurement on standard dataset for fair judgment. Finally their localization results are compared based on various error values and rank.
\end{abstract}

\section{Keywords}

Eye Localization, Performance Analysis.

\section{INTRODUCTION}

Eye localization is fundamental to all eyes tracking application. This task is different from other applications such as gaze estimation or blink detection. However fundamental to all these application is eye localization and the performance of all these applications will improve with robust eye localization[1]. The task of eye localization is difficult due to variations in appearance, facial expression, Occlusion, Pose, illumination, imaging quality and motion characteristics of an eye and may lead to a complex system design.

There are many intrusive techniques available for eye center localization that make use of electrooculography, Scleral coils, head mounted device or active infrared illumination. These techniques permit very accurate eye centers localization but they are not user friendly and less efficient in daylight and outdoor situations. Hence, it is necessary to study and analyze the efficiency of non-intrusive visible spectrum methods for eye center localization. These methods can be roughly divided as (i) Shape Based methods (ii) Feature-based methods, (iii) Appearance based Methods and (iv) Hybrid methods[2]. Detailed survey of video based eye detection and tracking methods is available at [2].

\section{EYE LOCALIZATION PERFORMANCE AND}

\section{EVALUATION CRITERIA}

To evaluate eye localization methods precisely and make a fair judgment among various methods, ideally the performance should be measured on benchmark database using standard error measure metrics, and follow a standard evaluation protocol[17]. However, in reality the algorithms are evaluated in different ways with variations in error measure metrics, databases, training samples, testing samples, etc., which makes it difficult to equally compare eye localization results[17]. A detail review on Efficient Performance Evaluation for Robust Eye Localization in terms of Error Measure Metric and Benchmark Databases with its characteristics and suitability is available at[1].

According to [1], the most commonly used error measurement is the normalized eye localization error proposed by Jesorsky[3] which is defined in terms of the eye center positions according to

$$
\text { e } \frac{1}{d} \max \left(e_{l}, e_{r}\right)
$$

where $d$ is the ground-truth position and $e_{1}$ and $e_{r}$ are the Euclidean distances between the detected eye centers and the ground-truths[17]. While analyzing the performance of various approaches for eye center localization, this measure has the following characteristics:

1 . $\mathrm{e} \leq 0.25 \approx$ Within eye center and the eye corners.

2. $\mathrm{e} \leq 0.10 \approx$ Within diameter of the iris.

3. $\mathrm{e} \leq 0.05 \approx$ Within diameter of the pupil[14].

Therefore, a good performance for $\mathrm{e} \leq 0.05$ is essential for precise eye center detection. Higher values of e are suitable for other general applications such as face detection, facial feature extraction etc.

According to [1], the BioID[4] dataset is found to be most appropriate and much more challenging for evaluation of eye localization as complex situation with real time environment is available in this dataset[1]

\section{METHODS}

In this section we briefly describe some methods, which to our knowledge are state-of-the art methods for eye localization. These methods adopt normalized error measurement proposed by Jesorsky[3] and use BioID[4] dataset for objective performance evaluation. In section 4, we evaluate the localization accuracy declared by these methods.

\subsection{Robust face detection using the Hausdorff distance}

Jesorsky[3] were among the first to highlight the performance and accuracy of the face detection. He proposed an eye localizer and a measure for quantitative evaluation for the performance of Eye Localization methods thru Hausdorff distance (HD), which is widely used nowadays. The Hausdorff distance (HD) is a metric between two point 
sets[3]. It is a relative error measure based on the distances between the expected and the estimated eye positions to validate the performance of eye detection system[3].

Their system adopts coarse to fine approach where task is divided into stage wise implementation as segmentation and localization stage to reduce the working portion of the image. In segmentation stage a face region is found and the edges are binarized and extracted. In localization stage, face position parameters are distinguished using previously built models, compared with edge images and the search is carried out for best match according to the Hausdorff distance [5]. The translation and scaling were also allowed in this approach. Finally a Multi-layer Perception (MLP) is trained with pupil centered images and the found positions are then refined to obtain accurate localization.

\subsection{Eye Localization using Face shape and appearance models}

Cristinacce[6] proposed a multi-stage approach for facial feature detection by combining the Boosted Cascade Face Detector[7], Pairwise Reinforcement of Feature Responses (PRFR) method for feature detection and an Active Appearance Model (AAM) for refinement of the predicted points.

Here, the face is located using Viola and Jones [7] boosted cascaded classifier. Each facial feature is then predicted from the detected face region using a detector. This detector is developed in their lab for various facial features using a manually labeled training set consisting of images. A Pairwise Reinforcement of Feature Responses (PRFR) method is proposed that models shape by learning the pairwise distribution of all true feature locations relative to the best match of each individual feature detectors[6]. The location of each feature is predicted by multiple detectors. The multiple predictions from multiple detectors for each feature point are then combined to make final prediction more robust. The predicted points are then refined using the Active Appearance Model (or AAM) to predict precise feature locations.

\subsection{Feature-based affine invariant localization of faces}

Hamouz[8] proposed a feature-based method that involves high resolution images of frontal faces. This method is applied stage wise as feature detection, Face Hypothesis Generation, Registration (scale and orientation normalization) and Appearance Verification.

The feature detection searches for 10 points (features) on the face by applying bank of Gabor filters. Gabor feature matrix is used to achieve scale and orientation invariance. Gaussian mixture model (GMM) is then used to capture appearance variations over a whole set of images to provide the best convergence properties and classification results. This process may generate false positive, hence refinement is done thru face hypothesis generation, registration, and appearance verification. Face hypothesis generation will consider each triplet of features and accept only geometrical feature configurations. Registration will apply affine transformation to the remaining candidate configurations for scale and rotation normalization. Finally SVM in coarse to fine manner is applied for verification step.

\subsection{D Cascaded AdaBoost for eye localization}

Niu [9] proposed a method based on AdaBoost classifier using both positive and negative examples similar to Viola Jones[7]. This method (1) Facilitates the classifier design on huge-scale training set (2) Deal with the significant variations within the positive or negative samples (3) Provide efficient training and testing procedures.

The first classifier is built based on randomly chosen small set of positive examples and progressively commencing the negative ones, which subsequently reduces the false detection rate. The entire process is repeated $n$ times to determine the increasing discrimination capacity of $\mathrm{n}$ classifiers. However this will also increase time consumption as last classifier will use thousands of Haar features. Localization is performed by two methods - one by weighting all classifiers and the other cascading them. The first is effective, but time consuming. The second one is faster and achieves high accuracy by obtaining the classifiers in cascade.

\subsection{Eye Localization using Edge Projections}

T"urkan[10] proposed an eye localization algorithm for faces with front pose and straight orientation. A face is detected first using edge projections method[10]. After defining a face candidate region, a single-stage 2-D rectangular wavelet transform of each region is computed and wavelet domain sub-images are obtained. This way, edges of the region are highlighted, and a caricature like representation is obtained[10]. The low-high and high-low sub-images will contain horizontal and vertical edges of the region, respectively. If the face region is sharp, the high-high sub image may contain almost all the edges.

The candidate points for each eyes are detected by analyzing horizontal projections and profiles of edge regions in the highpass filtered image. Support vector machine (SVM) based classifier is then used to estimate most feasible candidate points.

\subsection{Eye Center Localization using Isophote Curvature}

Valenti[11] proposed a method to accurately locate eye centers in low-resolution videos and images. It makes use of isophote properties to achieve invariance to lighting and rotation with low computational costs. The isophotes of an image are curve connecting points of equal intensity[11]. It is a contour lines not intersecting each other, acquired by slicing the intensity landscape with horizontal planes. The shape of the isophotes is independent to rotation and linear lighting changes. Since the curvature is proportional to radius, the radius of the circle is obtained from the curvature of the isophote. This curvature is combined with orientation and direction. The orientation can be estimated from the gradient but the direction of the gradient will be positive for brighter sclera rather than darker pupil. Therefore, the gradient is multiplied with the inverse of isophote curvature to obtain direction of the center.

Convolving the image with a Gaussian kernel, can boost the voting of a center and increase the number of isophotes around the edges. A center voting mechanism is used to weight important votes to boost the center estimates. 


\subsection{Pupil center Detection using Face Detection and CDF Analysis}

Asadifard[12] presented an adaptive eye localization algorithm that finds pupil center under varying expression and lighting conditions based on cumulative density function (CDF) framework.

The viola-Jones algorithm is used for real-time face detection. A search space is reduced by finding the region of interest (ROI) that is the area, that contains eye. This will substantially reduce the computational cost. The ROI is found by first dividing the face region vertically into top and bottom. Then the top part is further divided vertically into left and right segment that each segment contains an eye. The CDF is found by integrating the histogram of each of the ROI. Each ROI is then filtered by the cumulative distributed function (CDF) of that region. Next the erosion is applied on the resulting image and the minimum intensity pixel is determined. The average pixel intensity is calculated in a region of size $10 * 10$ and erosion is then applied on the original eye region. Pupil center is then determined in the region of size $15 * 15$.

\subsection{Eye Localization through Multi Scale Sparse Dictionaries}

Yang[13] proposed an eye localization method based on Multiscale Sparse Dictionaries (MSD). Here, pyramids of dictionaries are built to model context information at multiple scales by aligning training images using eye centers. There are separate dictionaries for both left and right eyes. Dictionary at each scale is built to acquire context information of specific area. Large context is useful for the variation of eye appearances, where as small context facilitates accurate eye localization.

The localization algorithm starts with largest context where eye location is estimated. Gradually smaller region with subsequent dictionaries are used to derive a closer eye location. This way by applying dictionaries from the largest to smallest scale, the estimated eye locations will gradually converge to accurate position. This method is efficient and resistant to image noises, however it is computationally expensive as eye locations are estimated with an iterative procedure.

\subsection{Eye Centre Localization by Means of Gradients}

Fabian[14] Proposed a multi stage approach for eye center localization using image gradients. This method is simple and invariant to changes in scale, pose, illumination and rotation. In Multi stage approach a face detector is applied first and rough eye location are extracted. This rough eye location is then used to derive precise estimation in next stage.

The center of a circular object can be detected by analyzing the vector field of image gradients. Fabian[14] derived a simple objective function, which only consists of dot products. For every pixel, the squared dot product between the displacement vector of a center candidate and the image gradient is computed. The position of the maximum then corresponds to the position where most image gradients intersect which is ideally the eye's center. Considering only gradient vectors with a significant magnitude the computational complexity can be decreased. A weight is applied for each possible center to avoid wrong center estimation due to dominant eyelids, eyelashes, wrinkles and a low contrast between iris and sclera. The image is smoothed by a Gaussian filter to avoid problems of reflections such as glasses. The proposed summation of weighted squared dot products yields accurate results if the image contains the eye[14].

\subsection{Eye localization for low quality face images}

In low quality images for eye localization, it is difficult to discriminate between the positive and negative samples. The positive low quality samples are many times rejected by the threshold in the cascade and fail to contribute to the final result[15]. Yi[15] proposed an eye localization method for low quality face images to improve the eye detection rate and localization precision.

Yi[15] proposed a probabilistic cascade (P-Cascade) framework, where the traditional cascade classifier is reformulated in a probabilistic way. In P-Cascade all image patches are treated equally and each image patch contribute to the final result, irrespective the samples accepted or rejected by the cascade. This way, P-Cascade can adapt well to various image quality. Further two extensions are proposed to improve the precision and robustness of P-Cascade (1) Extending LBP (ELBP) feature set (2) Stacking classifiers in multiple scales.

Yi[15] introduced ELBP (Extended LBP) as an improvement to LBP and multi block LBP (MB-LBP) to enhance the classification ability and improve the system's precision by enhancing the discriminant of basic features. Two classifiers in multiple scales are used to increase robustness and efficiency. The first classifier excludes negative pattern similar to eyes, while second classifier covers only the eye region to achieve high localization precision robustly.

\subsection{Eye Localization Method with Rotation Invariance}

Ren[16] proposed a method to overcome the difficulty of precise eye localization in arbitrary rotation conditions. The problem is addressed by adopting the invariant feature-based codebook, integrating the sparse representation classifier method with the Pyramid-like localizing strategy and applying more prior information into the framework.

The method consists of three stages. Firstly, a Codebook of Invariant Local Features is established which contains feature based structural information of eyes which is then used to characterize eye and non eye samples. Next, a 2-class sparse representation classifier (SRC) with multivalued output integrated with a novel Pyramid-like detecting and locating method is proposed to preserve the rotation invariance in classification and localization steps in a common framework. Traditional sliding window method is used in prevailing methods for eye localization where local search window is moved from pixel to pixel to find position of an object. For large search space and rotational changes, this approach is inappropriate. A novel Heat Map by Pyramid-like detecting and locating method is used to preserve the invariant property. Some prior knowledge of color images is combined with the output of SRC classifier, which is sensitive of eye positions and invariant of eye rotations.

\section{ANALYSIS OF METHODS}

The existing published results of state of the Art methods viz. Jesorsky[3], Cristinacce[6], Hamouz[8], Niu[9], T"urkan[10], Valenti[11], Asadifard[12], Yang[13], Fabian[14], Yi[15], Ren[16] are analysed. All these methods have been assessed for Normalized Error Measurement proposed by Jesorsky[3] and tested on BIOID dataset[4]. The performance evaluation 
for e $\in\{0.05,0.10,0.15,0.20,0.25\}$ of these methods are required to be measured to justify fair evaluation. However in many cases, the performance for all error values are not mentioned by the respective authors, and only few error values are mentioned along with a worse eye Characteristic (WEC) curve.

These lacking error values from the respective authors graph are appropriately derived and indicated in bracket as shown in table 1. Figure 1 shows the Performance measurement for all method for various and individual values of e and overall performance of all methods. Table 2 indicates top 5 performing methods for each error value. Table 3 shows performance comparison of methods based on rank which is roughly inversely proportional to the area under WEC as proposed by Fabian[14]. Figure 2 shows the Performance measurement for all method based on rank values and overall rank of all methods. Here, the performance of $\mathrm{e} \leq 0.05$ for various methods is very essential as this value indicates efficient eye localization where as, the performance of higher value of $\mathrm{e}$ is suitable for other application such as face detection, facial feature analysis etc.

\subsection{Performance Analysis based on Normalized Error Value and Rank Eye center localization (for $e \leq 0.05)$ :}

1. Method proposed by Yang[13] yields best performance of $89.60 \%$ which is highest in this category and which is also the essential condition for accurate eye localization. However, this method is highly iterative and computationally expensive as it uses Multiscale Sparse Dictionaries and K-SVD Dictionary Compression,

2. Methods proposed by Yi[15], Valenti [11] and Ren[16] yield 2nd, 3rd, 5th best performance respectively as seen in table 2. All these methods use some kind of machine learning techniques, hence computationally complex.

3. Fabian[14] method holds 4 th position with reasonably good performance of $82.5 \%$ for e $\leq 0: 05$ which is $7.1 \%$ lower to Yang[13], fairly close to Yi[15] (4\%) and Valenti[11] (1.6\%) and superior to Ren[16]. It has significantly less computational complexity, as it does not require any iteration, clustering or classifier.

Eye center localization ( $e \leq 0.10$ and $e \leq 0.15)$ :

1. Yi[15] provides the best performance of $99.1 \%$ and $99.3 \%$ for both $\mathrm{e} \leq 0.10$ and $\mathrm{e} \leq 0.15$ respectively with Extending LBP (ELBP) feature set, Stacking classifiers in multiple scales and P-Cascade.

2. Next best performance for $\mathrm{e} \leq 0.10$ is achieved from Cristinacce[6] and Yang[13] (96.0\% \& 95.5\% respectively) and from Yang[13] and Cristinacce[6] for e $\leq 0.15$ (97.2 \& 96.5\% respectively) with complex methods.

Table 1 : Performance Comparison of various methods using Normalized Error Measurement using the BioID database.

\begin{tabular}{|l|l|c|c|c|c|c|l|}
\hline $\begin{array}{l}\text { Sr } \\
\text { No }\end{array}$ & Method & $\mathbf{e} \leq \mathbf{0 . 0 5}$ & $\mathbf{e} \leq \mathbf{0 . 1 0}$ & $\mathbf{e} \leq \mathbf{0 . 1 5}$ & $\mathbf{e} \leq \mathbf{0 . 2 0}$ & $\mathbf{e} \leq \mathbf{0 . 2 5}$ & Remarks \\
\hline 1 & Jesorsky[3] & $(38.5 \%)$ & $(79.0 \%)$ & $(84.5 \%)$ & $(87.0 \%)$ & $91.8 \%$ & Multi-layer perceptron (MLP) Localizer \\
\hline 2 & Cristinacce[6] & $(57.0 \%)$ & $96.0 \%$ & $(96.5 \%)$ & $(97.0 \%)$ & $(97.1 \%)$ & $\begin{array}{l}\text { Pairwise Reinforcement Feature Responses } \\
\text { (PRFR) } \\
\text { Active Appearance model (AAM) }\end{array}$ \\
\hline 3 & Hamouz[8] & $(59.0 \%)$ & $(75.0 \%)$ & $(80.8 \%)$ & $(87.6 \%)$ & $(91.0 \%)$ & $\begin{array}{l}\text { Gaussian mixture model (GMM) } \\
\text { Support Vector Machines (SVM) }\end{array}$ \\
\hline
\end{tabular}

3. Fabian[14] method, which is solely based on dot products, achieves the 4 th and 5 th best performance for $\mathrm{e} \leq 0.10$ and $\mathrm{e}$ $\leq 0.15$ (93.4\% \& $95.2 \%$ respectively) which is only $5.7 \%$ and $4.1 \%$ worse compared to Yi[15] which is the best method in this category.

Eye center localization ( $e \leq 0.20$ and $e \leq 0.25)$ :

1.For e $\leq 0.20 \mathrm{Yi}[15]$ achieves best performance of $99.5 \%$. For e $\leq 0.25 \mathrm{Yi}[15]$ and T"urkan[10] achieves $99.5 \%$ and $99.6 \%$ accuracy respectively. Both the methods are complex and involve some machine learning techniques.

2.Fabian[14] method performs with $96.4 \%$ and $98.0 \%$ accuracy for $\mathrm{e} \leq 0.20$ and $\mathrm{e} \leq 0.25$ respectively which is only $3.1 \%$ and $1.6 \%$ worse to the best method in this category without any complex techniques.

\subsection{Performance Analysis based on the rank:}

A comparison based on the rank of each method according to its performances in table 1 is shown in Table 3 and figure 2. Following are the analysis:

1. There is no single method that performs superior for all error values.

2. Method proposed by Yi[15] achieves best performance with reasonable complexity and ranks $1^{\text {st }}$ for all values of e except for $\mathrm{e} \leq 0.05$. However, here the higher performance especially for $\mathrm{e} \leq 0.05$ is very much desired for accurate eye localization.

3. Yang[13] achieves best performance only for e $\leq 0.05$ and performs reasonable for other error values. This method achieves $2^{\text {nd }}$ average rank but it is highly iterative and computationally very expensive.

4. Ren[16] achieves $3^{\text {rd }}$ position with average rank of 4.4 but the method performs poorly for $\mathrm{e} \leq 0.05$ and $\mathrm{e} \leq 0.10$.

5. Fabian[14] method achieves $4^{\text {th }}$ position with average rank of 4.6. It ranks 4 th for both pupil and iris localization ( $\mathrm{e} \leq$ 0.05 and $\mathrm{e} \leq 0.10$ ) and ranks 5 th for larger e.

6. Fabian[14] method ranks only 0.2 worse to Ren[16] which is $3^{\text {rd }}$ best method.

7. The variance of the individual ranks in Fabian[14] method is significantly less, and the complexity of this method is much lower.

8. Method proposed by Valenti[11] achieves $5^{\text {th }}$ rank. This method ranks $3^{\text {rd }}$ for $\mathrm{e} \leq 0.05$ but ranks poor for $\mathrm{e} \leq 0.10$ and $\mathrm{e} \leq 0.15$. 


\begin{tabular}{|l|l|c|c|c|c|c|l|}
\hline 4 & Niu [9] & $(75.0 \%)$ & $93.0 \%$ & $(95.8 \%)$ & $(96.4 \%)$ & $(97.0 \%)$ & $\begin{array}{l}\text { 2D cascade classifier, } \\
\text { Large training dataset. }\end{array}$ \\
\hline 5 & T"urkan [10] & $(18.6 \%)$ & $73.7 \%$ & $(94.2 \%)$ & $(98.7 \%)$ & $99.6 \%$ & $\begin{array}{l}\text { Wavelet transform, } \\
\text { SVM classifier }\end{array}$ \\
\hline 6 & Valenti [11] & $84.1 \%$ & $90.9 \%$ & $(93.8 \%)$ & $(97.0 \%)$ & $98.5 \%$ & $\begin{array}{l}\text { Isophote Curvature, } \\
\text { MIC+SIFT+kNN Learning }\end{array}$ \\
\hline 7 & Asadifard [12] & $47.0 \%$ & $86.0 \%$ & $89.0 \%$ & $93.0 \%$ & $96.0 \%$ & $\begin{array}{l}\text { Cumulative density func(CDF), * } \\
\text { \# No learning model require. }\end{array}$ \\
\hline 8 & Yang[13] & $89.60 \%$ & $95.50 \%$ & $(97.2 \%)$ & $(98.5 \%)$ & $99.10 \%$ & $\begin{array}{l}\text { Multiscale Sparse Dictionaries, } \\
\text { K-SVD Dictionary Compression, Highly } \\
\text { iterative method. }\end{array}$ \\
\hline 9 & Fabian[14] & $82.5 \%$ & $93.4 \%$ & $95.2 \%$ & $96.4 \%$ & $98.0 \%$ & $\begin{array}{l}\text { Gradient, squared dot products } \\
\text { \# No learning model require. }\end{array}$ \\
\hline 10 & Yi[15] & $86.5 \%$ & $99.1 \%$ & $(99.3 \%)$ & $(99.5 \%)$ & $(99.6 \%)$ & S1+S2, E-LBP, P-Cascade \\
\hline 11 & Ren[16] & $77.08 \%$ & $92.25 \%$ & $(95.7 \%)$ & $(97.0 \%)$ & $98.99 \%$ & $\begin{array}{l}\text { Scale-invariant feature transform (SIFT), } \\
\text { 2-class sparse representation classifier (SRC) }\end{array}$ \\
\hline
\end{tabular}

() Brackets indicate values derived from respective author's graph. * Images with glasses and closed eyes were omitted.

\# Methods don't involve any learning or model scheme.

Table 2 : Top 5 performing methods for various Normalized Error

\begin{tabular}{|c|c|c|c|c|c|}
\hline Rank & $\mathbf{e} \leq \mathbf{0 . 0 5}$ & $\mathbf{e} \leq \mathbf{0 . 1 0}$ & $\mathbf{e} \leq \mathbf{0 . 1 5}$ & $\mathbf{e} \leq \mathbf{0 . 2 0}$ & $\mathbf{e} \leq \mathbf{0 . 2 5}$ \\
\hline 1 & $89.60 \%[13]$ & $99.1 \%[15]$ & $(99.3 \%)[15]$ & $(99.5 \%)[15]$ & $(99.6 \%)[15][10]$ \\
\hline 2 & $86.5 \%[15]$ & $96.0 \%[6]$ & $(97.2 \%)[13]$ & $(98.7 \%)[10]$ & $99.10 \%[13]$ \\
\hline 3 & $84.1 \%[11]$ & $95.50 \%[13]$ & $(96.5 \%)[6]$ & $(98.5 \%)[13]$ & $98.99 \%[16]$ \\
\hline 4 & $82.5 \%[14]$ & $93.4 \%[14]$ & $(95.8 \%)[9]$ & $(97.0 \%)[6][11][16]$ & $98.5 \%[11]$ \\
\hline 5 & $77.08 \%[16]$ & $93.0 \%[9]$ & $95.2 \%[14]$ & $96.4 \%[14][9]$ & $98.0 \%[14]$ \\
\hline
\end{tabular}

Table 3: Comparison of ranks of each method according to its performance

\begin{tabular}{|c|c|c|c|c|c|c|c|}
\hline $\begin{array}{c}\text { Sr } \\
\text { No }\end{array}$ & Method & $\mathbf{e} \leq \mathbf{0 . 0 5}$ & $\mathbf{e} \leq \mathbf{0 . 1 0}$ & $\mathbf{e} \leq \mathbf{0 . 1 5}$ & $\mathbf{e} \leq \mathbf{0 . 2 0}$ & $\mathbf{e} \leq \mathbf{0 . 2 5}$ & $\begin{array}{c}\text { Avg. } \\
\text { Rank }\end{array}$ \\
\hline 1 & Jesorsky[3] & 10 & 9 & 9 & 8 & 9 & 9 \\
\hline 2 & Cristinacce[6] & 8 & 2 & 3 & 4 & 6 & 4.6 \\
\hline 3 & Hamouz[8] & 7 & 10 & 10 & 7 & 10 & 8.8 \\
\hline 4 & Niu [9] & 6 & 5 & 4 & 5 & 7 & 5.4 \\
\hline 5 & T̈urkan [10] & 11 & 11 & 6 & 2 & 1 & 6.2 \\
\hline 6 & Valenti [11] & 3 & 7 & 7 & 4 & 4 & 5 \\
\hline 7 & Asadifard [12] & 9 & 8 & 8 & 6 & 8 & 7.8 \\
\hline 8 & Yang[13] & 1 & 3 & 2 & 3 & 2 & 2.2 \\
\hline 9 & Fabian[14] & 4 & 4 & 5 & 5 & 5 & 4.6 \\
\hline 10 & Yi[15] & 2 & 1 & 1 & 1 & 1 & 1.2 \\
\hline 11 & Ren[16] & 5 & 6 & 4 & 4 & 3 & 4.4 \\
\hline
\end{tabular}




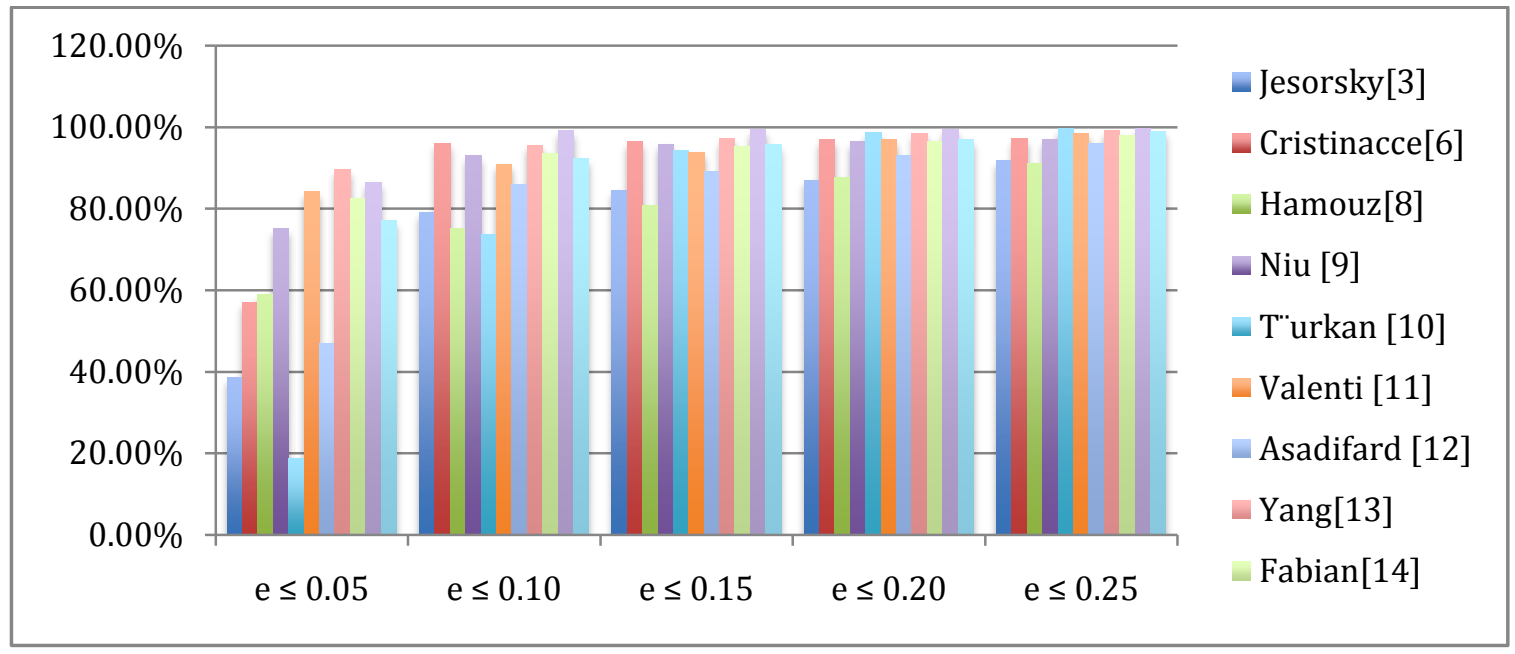

(a)

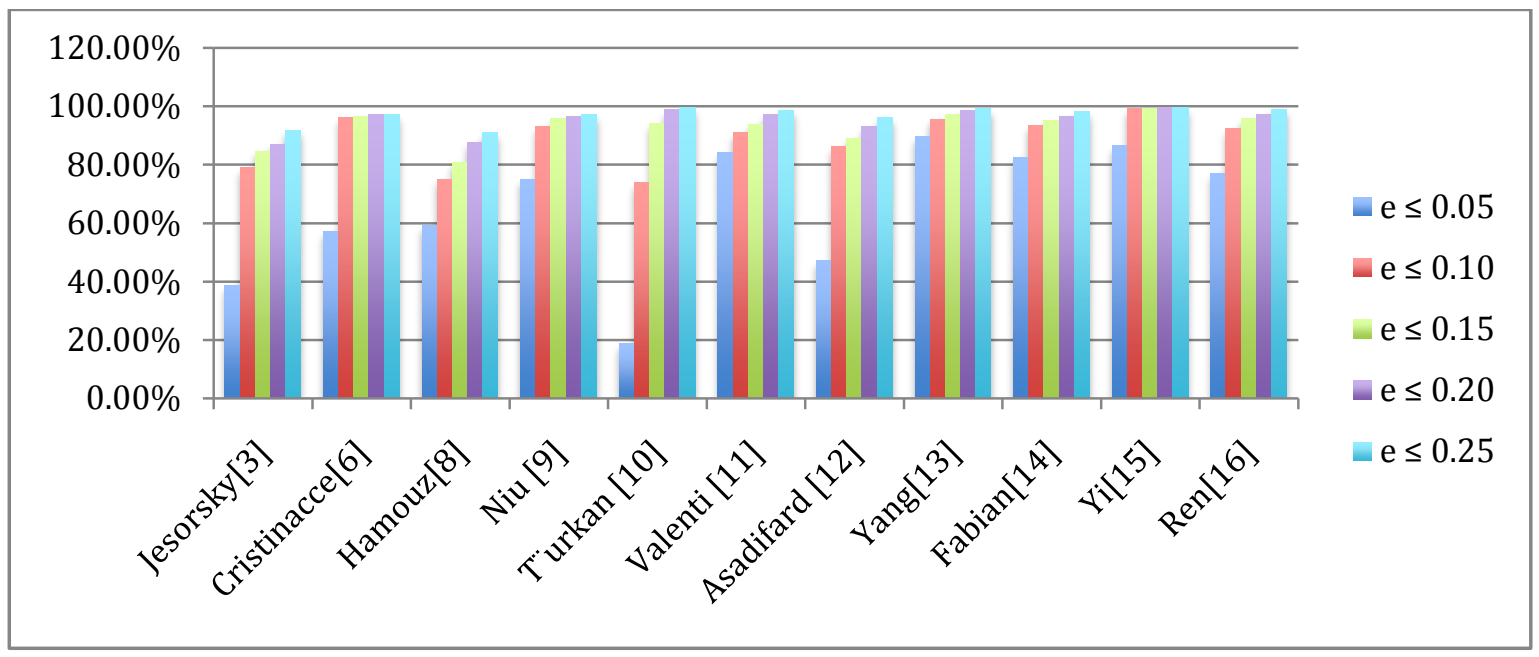

(b)

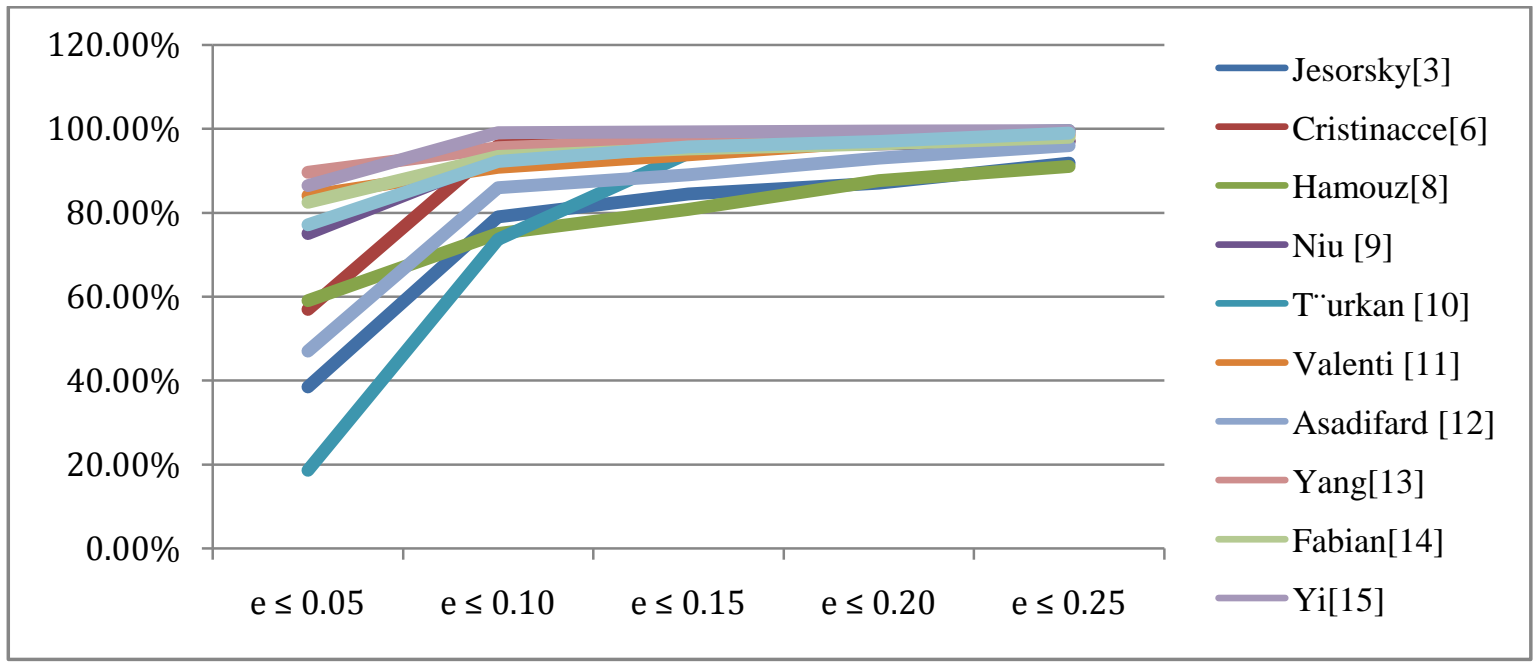

(c)

Figure 1 : (a) Performance measurement for all method for individual values of e. (b) Performance measurement of individual method for various values of e. (c) Overall performance of individual methods. 


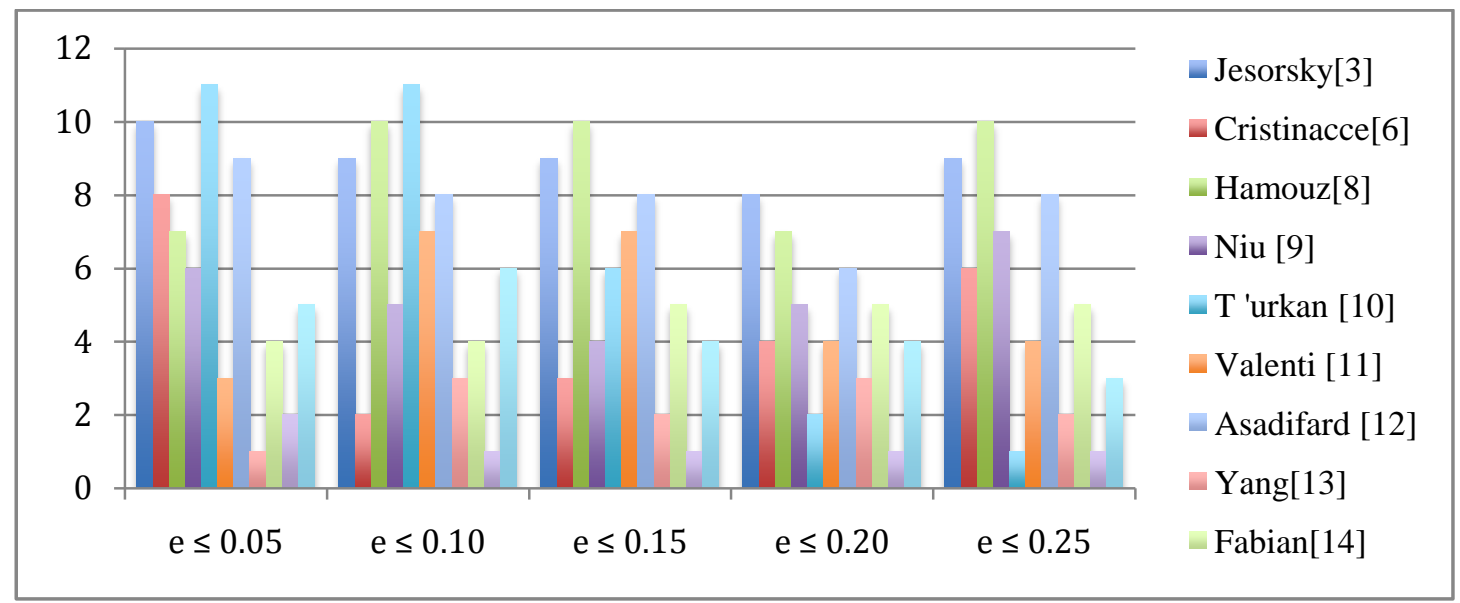

(a)

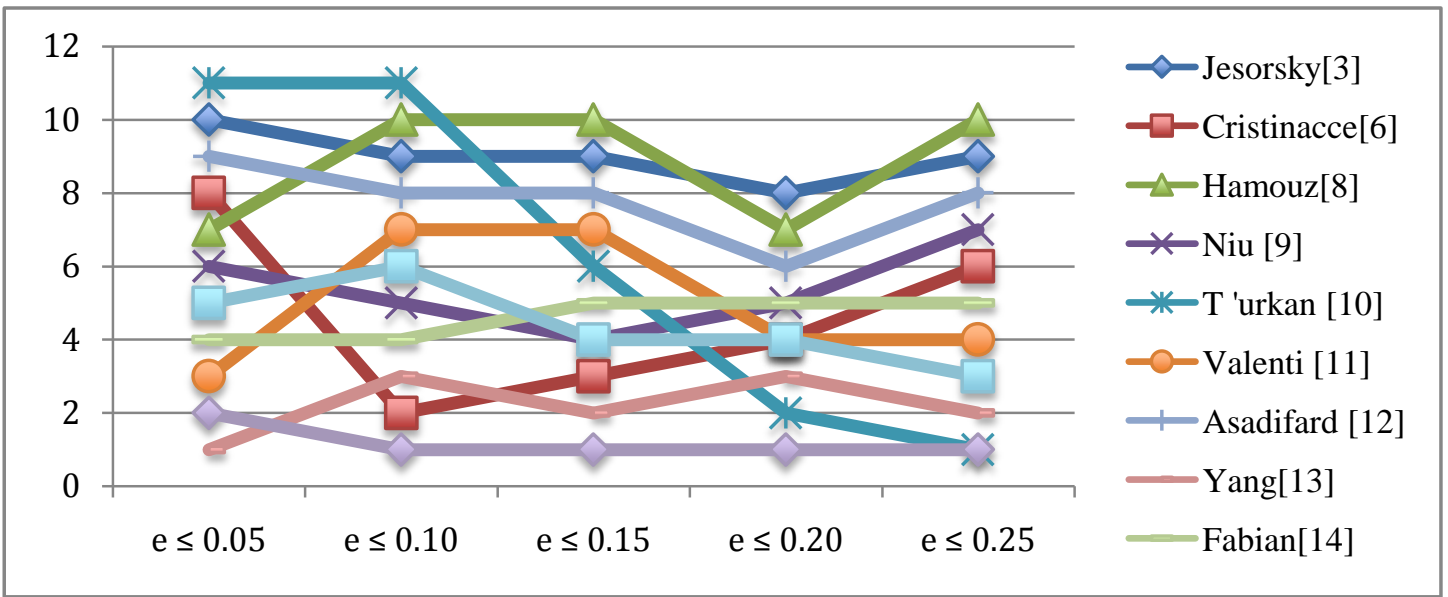

(b)

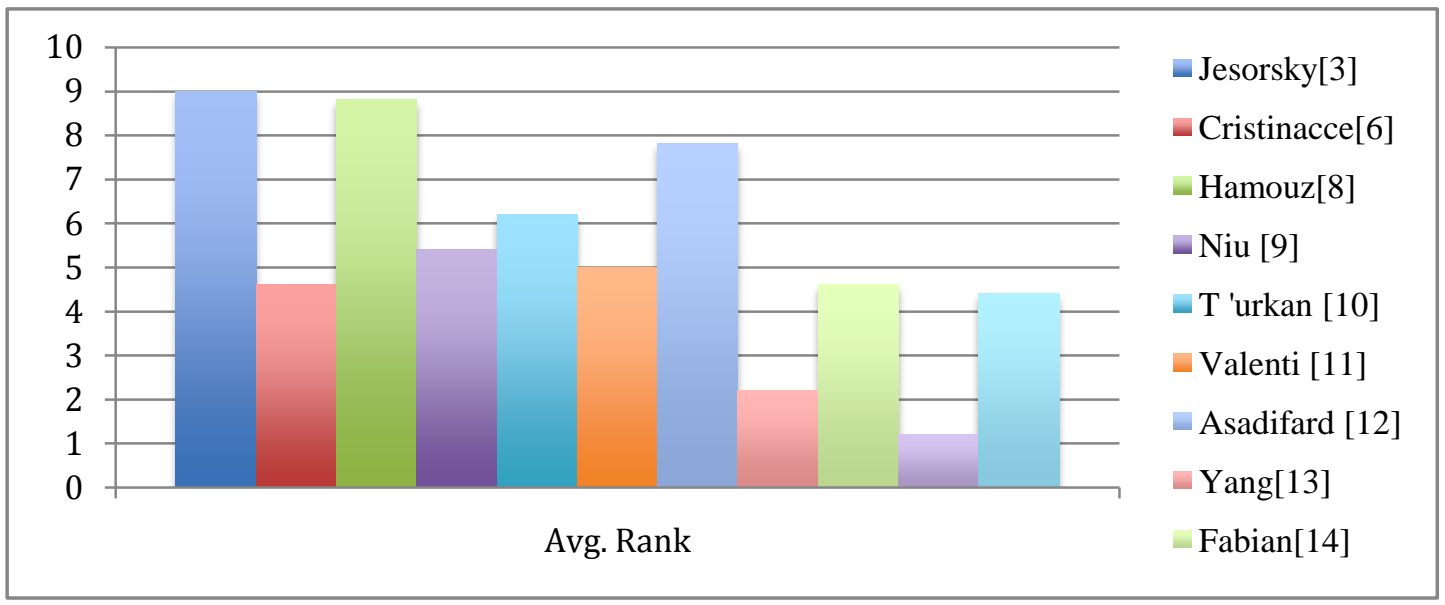

(c)

Figure 2 : Comparison of ranks (a) Performance measurement on rank for all method for individual values of e. (b) Rank of each method on various values of e (c) Overall rank of individual methods.

\section{CONCLUSION}

Efficient eye localization is a step stone for all eye tracking applications. It is therefore necessary to evaluate the efficiency of existing eye localization techniques to derive best suitable method. The state-of-the-art eye localization methods are reviewed briefly that uses normalized error measurement on BioID database for fair performance evaluation. Existing available results provided by the respective authors of various methods are compared for analysis. In many cases, the authors do not mention all the values of normalized error for the performance analysis, then the appropriate lacking error values are derived from the respective authors graph and used for evaluation. The rank of all methods for individual error value and average rank for all methods are also derived. The 5 best performing methods according to its average rank are identified which are 
proposed by Yi[15], Yan[13], Ren[16], Fabian[14] and Valenti [11] respectively.

The methods proposed by Yi[15], Yan[13], Ren[16] ranks as top 3 methods respectively according to average rank and claim to have higher accuracy but they are complex, require some machine learning techniques and computationally expensive. The method proposed by Fabian[14] ranks $4^{\text {th }}$ according to average rank but it is efficient and computationally less complex as only dot products are involved and no machine learning techniques are required. The variance of Fabian[14] method is significantly less for all error values. Method proposed by Valenti[11] achieves $5^{\text {th }}$ average rank. Therefore if the accuracy is the most concern, the method proposed by Yi[15] is a suitable choice which also has a reasonable complexity but if a less complexity is the concern, the method proposed by Fabian[14] is most appropriate method with an equitable accuracy for a wide range of eye tracking applications.

\section{REFERENCES}

[1] K N Jariwala, U D Dalal, Efficient Performance Evaluation for Robust Eye Localization System, International Journal of Computer Science Trends and Technology (IJCST), Volume 3 Issue 1, Jan-Feb 2015.

[2] Hansen, D. and Ji, Q, In the eye of the beholder: A survey of models for eyes and gaze. IEEE Trans. on PAMI, 32(3):478-500, 2010

[3] O. Jesorsky, K.J. Kirchberg, and R.W. Frischholz. Robust face detection using the hausdorff distance. Lecture Notes in Computer Science, LNCS2091 pp 9095, June 2001

[4] www.bioid.com/About/BioID-Face-Database

[5] P.Campadelli, R.Lanzarotti, G.Lipori, Eyelocalization : a survey, in: The Fundamentals of Verbal and Non-verbal Communication and the Biometrical Issue NATO Science Series, vol.18, pp.234-245, 2007.

[6] Cristinacce, D., Cootes, T., and Scott, I. (2004). A multistage approach to facial feature detection. In Proceedings of the 15th BMVC, pages 277-286, England.

[7] Viola, P. and Jones, M, Robust real-time face detection. IJCV, 57(2): 137-154, 2004.
[8] M. Hamouz, J. Kittler, J.K. Kamarainen, P. Paalanen, H. Kälviäinen, and J. Matas. Feature-based affine invariant localization of faces. IEEE Trans. Pattern Analysis and Machine Intelligence, 27(9):1490- 1495, 2005.

[9] Z. Niu, S. Shan, S. Yan, X. Chen, and W. Gao. 2D Cascaded AdaBoost for Eye Localization. Proc. Of the 18th Internationl Conference on Pattern Recognition, 2006.

[10] T"urkan, M., Pard as, M., and Cetin A., Human eye localization using edge projections. In Proceedings of the VISAPP, pages 410-415, 2007.

[11] Valenti, R. and Gevers, T., Accurate eye center location and tracking using isophote curvature. In Proceedings of the CVPR, pages $1-8,2008$.

[12] Asadifard, M. and Shanbezadeh, J., Automatic adaptive center of pupil detection using face detection and cdf analysis. In Proceedings of the IMECS, volume I, pages 130-133, 2010

[13] Fei Yang, Junzhou Huang, Peng Yang and Dimitris Metaxas, Eye Localization through Multiscale Sparse Dictionaries, IEEE International Conference on Automatic Face and Gesture Recognition, Santa Barbara, March. 2011

[14] F. Timm and E. Barth, "Accurate eye centre localisation by means of gradients," in Proc. VISAPP, pp. 125-130, March 2011.

[15] D. Yi, Z. Lei, and S. Z. Li, "A robust eye localization method for low quality face images," in Proceedings of the International Joint Conference on Biometrics (IJCB '11), pp. 1-6, Washington, DC, USA, October 2011.

[16] Yan Ren, Shuang Wang, Biao Hou, Jingjing Ma, A Novel Eye Localization Method With Rotation Invariance, IEEE Transactions On Image Processing, VOL. 23, NO. 1, January 2014.

[17] Fengyi Song, Xiaoyang Tan, Songcan Chen, Zhi-Hua Zhou, A literature survey on robust and efficient eye localization in real-life scenarios, Pattern Recognition Volume 46, Issue 12, Pages 3157-3173 December 2013. 